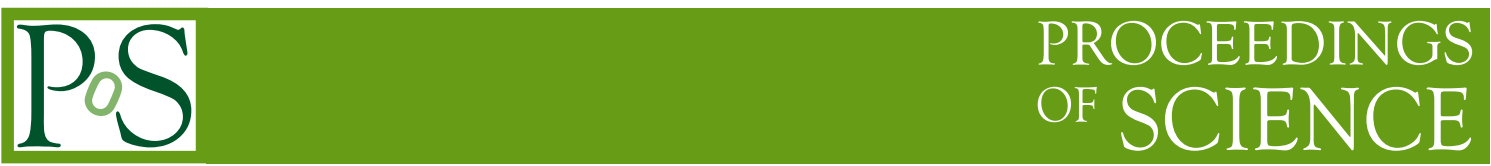

\title{
Gravitational Lensing in Plasma
}

\author{
O.Yu. Tsupko ${ }^{a b}$ and G.S. Bisnovatyi-Kogan ${ }^{a b}$ \\ ${ }^{a}$ Space Research Institute of Russian Academy of Sciences, \\ Profsoyuznaya 84/32, Moscow 117997, Russia \\ ${ }^{b}$ National Research Nuclear University MEPhI, \\ Kashirskoe Shosse 31, Moscow 115409, Russia \\ E-mail: tsupko@iki.rssi.ru, gkogandiki.rssi.ru
}

We develop a model of gravitational lensing in plasma. We show that the gravitaional deflection angle by point mass in the homogeneous plasma differs from the vacuum deflection angle and depends on frequency of the photon. The dependence of the lensing angle on the photon frequency in the homogeneous plasma resembles the properties of a refractive prism spectrometer, the strongest action of which is for very long radio waves. We also derive the photon deflection by point mass in the non-homogeneous plasma. Then we consider more general case of the photon deflection by mass distibution in non-homogeneous plasma. Our approach allows us consider two effects simultaneously: the gravitational deflection in plasma which differs from the vacuum case, and the non-relativistic effect (refraction) connected with the plasma inhomogeneity.

25th Texas Symposium on Relativistic Astrophysics

December 6-10, 2010

Heidelberg, Germany 


\section{Introduction}

The photon deflection angle in vacuum, in the Schwarzschild metric with a given mass $M$, is determined, for small deflection angles $\hat{\alpha} \ll 1$, by a formula

$$
\hat{\alpha}=\frac{4 G M}{c^{2} b}=\frac{2 R_{S}}{b},
$$

where $b$ is the impact parameter, and $b \gg R_{S}, R_{S}=2 G M / c^{2}$ is the Schwarzschild radius [1], 2]. On basis of Einstein deflection angle ordinary gravitational lensing theory is developed [2, 3, 4]. Angle (1.1) does not depend on frequency of the photon, so gravitational lensing in vacuum is achromatic.

The photon deflection in a non-homogeneous plasma, in presence of gravity, has been considered by different authors [4, 5, 6]. The consideration was done in a linear approximation. Total photon deflection was considered as a sum of the two effects: the vacuum deflection due to the gravitation, and the deflection due to the non-homogeneity of the medium (refraction). In this works these two effects were considered separately. The first effect is achromatic, the second one depends on the photon frequency if the medium is dispersive, but equals to zero if the medium is homogeneous. So in this approximation the deflection angle in the homogeneous plasma did not differ from the Einstein deflection angle.

A general theory of the geometrical optic in the curved space-time, in arbitrary medium, is presented in the book of Synge [7]. On the basis of his general approach we have developed the model of gravitational lensing in plasma. In our work [8] we have shown for the first time, that in a nonlinear approach a new effect appears. Due to dispersive properties of plasma even in the homogeneous plasma the gravitational deflection angle differs from the vacuum deflection angle and depends on frequency of the photon. In next work [9] we derive the deflection angle for the photon moving in a weak gravitational field, in the arbitrary inhomogeneous plasma. Our approach allows us consider two effects simultaneously for different gravitational lens model: the gravitational deflection in plasma and the non-relativistic effect (refraction) connected with the plasma inhomogeneity. Self-consistent approach for the light deflection in presence of the gravitation and the plasma is also developed in book of Perlick [10].

\section{Gravitational deflection by point mass in homogeneous plasma. Gravitational radiospectrometer.}

Let us consider a static space-time with the Schwarzschild metric of the point mass $M$ and assume that the gravitational field is weak. Let us consider, in this gravitational field, a static inhomogeneous spherically-symmetric plasma, with the concentration $N=N(r)$, and we do not assume that $N(\infty)=0$. A refraction index $n$ of plasma is:

$$
n^{2}=1-\frac{\omega_{e}^{2}}{[\omega(r)]^{2}}, \quad \omega_{e}^{2}=\frac{4 \pi e^{2} N(r)}{m}=K_{e} N(r) .
$$

Here $\omega(r)$ is the frequency of the photon, which in spherically symmetric case depends on the space coordinate $r$ due to the presence of the gravitational field (gravitational red shift). We denote $\omega(\infty) \equiv \omega, e$ is the charge of the electron, $m$ is the electron mass, $\omega_{e}$ is the electron plasma frequency. The impact parameter $b$ remains constant in the null approximation for the photon moving 
along the axis $z$. The position of the photon is characterized by $b$ and $z$, and the absolute value of the radius-vector is $r=\sqrt{b^{2}+z^{2}}$. In this model the plasma is just the medium with given refraction index, we neglect the self-gravitation of the plasma particles, compared to the gravity of a central massive object.

We have derived the expression for the deflection angle of the photon moving in a weak gravitational field with the Schwarzschild metric, in a homogeneous plasma with constant $N$ and $\omega_{e}$ [8], [9]:

$$
\hat{\alpha}=\frac{R_{S}}{b}\left(1+\frac{1}{1-\left(\omega_{e}^{2} / \omega^{2}\right)}\right),
$$

which turns into the expression (1.1), when $\omega \rightarrow \infty$. The effect of difference in the gravitational deflection angles is significant for longer wavelengths, when $\omega$ is approaching $\omega_{e}$, what is possible only for the radio waves. Therefore, the gravitational lens in plasma acts as a gravitational radiospectrometer [8], [9].

In an observation of two images of the lensing point source in presence of homogeneous plasma, the following features may be observed: spectra of two images may be different in the long wave side due to different plasma properties along the trajectories of light rays forming the images, the extended image may have different spectra in different parts of the image, with a maximum of the spectrum shifting to the long wave side in the regions with a larger deflection angle [9].

We have shown that in the homogeneous plasma gravitational deflection angle differs from vacuum deflection angle and depends on frequency of the photon. In the paper of Kulsrud \& Loeb [11] (see also [12], [13]) it was shown that in a homogeneous plasma the photon wave packet moves like a particle with a velocity equal to the group velocity of the wave packet, and with a mass equal to the plasma frequency. In work [9] we have shown that our result for a homogeneous plasma follows also from this approach.

The difference of gravitational deflection in a homogeneous medium is only if the medium is dispersive. Let us consider the homogeneous medium without dispersion, with the refraction index $n=$ const $\geq 1$ not depending on the photon frequency $\omega$. In paper [8] we have shown that in the case of a non-dispersive medium, the constant refraction index is cancelled, and the photon trajectory is the same as in vacuum, in the presence of a gravitational field, in spite of lower velocity of light propagation in the medium. Note that the motion of photons in 4-space, in the medium, is not described by the geodesic equation (neither massive nor zero-mass) because light propagation in a medium with refraction is determined not only by the gravitational field but also by the medium.

\section{Photon deflection by point mass in non-homogeneous plasma.}

We derive the deflection angle for the photon moving in a weak gravitational field, in the Schwarzschild metric of point mass, in the arbitrary inhomogeneous plasma:

$$
\hat{\alpha}_{b}=\frac{R_{S}}{b}+\int_{0}^{\infty}\left(\frac{1}{1-\left(\omega_{e}^{2} / \omega^{2}\right)} \frac{R_{S} b}{r^{3}}+\frac{K_{e}}{\omega^{2}-\omega_{e}^{2}} \frac{b}{r} \frac{d N(r)}{d r}\right) d z .
$$

Approximation is that the whole deflection angle, from the combined plasma and gravity effects, remains small [9]. To demonstrate the physical meaning of different terms in (3.1], we write this 
expression under condition $1-n=\omega_{e}^{2} / \omega^{2} \ll 1$. Carrying out the expansion of terms with the plasma frequency, we obtain:

$$
\hat{\alpha}_{b}=\frac{2 R_{S}}{b}+\frac{R_{S} b}{\omega^{2}} \int_{0}^{\infty} \frac{\omega_{e}^{2}}{r^{3}} d z+\frac{K_{e} b}{\omega^{2}} \int_{0}^{\infty} \frac{1}{r} \frac{d N(r)}{d r} d z+\frac{K_{e} b}{\omega^{4}} \int_{0}^{\infty} \frac{\omega_{e}^{2}}{r} \frac{d N(r)}{d r} d z .
$$

The first term is a vacuum gravitational deflection. The second term is an additive correction to the gravitational deflection, due to the presence of the plasma. This term is present in the deflection angle both in the inhomogeneous and in the homogeneous plasma, and depends on the photon frequency. The third term is a non-relativistic deflection due to the plasma inhomogeneity (the refraction). This term depends on the frequency, but it is absent if the plasma is homogeneous. The forth term is a small additive correction to the third term.

\section{Photon deflection by mass distibution in non-homogeneous plasma.}

In case of gravitational lensing by mass distribution we have the formula:

$$
\hat{\alpha}_{b}=\frac{4 G M(b)}{c^{2} b}+\frac{2 G M(b) b}{c^{2} \omega^{2}} \int_{0}^{\infty} \frac{\omega_{e}^{2} d z}{r^{3}}+\frac{K_{e} b}{\omega^{2}} \int_{0}^{\infty} \frac{d N(r)}{d r} \frac{d z}{r}+\frac{K_{e} b}{\omega^{4}} \int_{0}^{\infty} \frac{d N(r)}{d r} \frac{\omega_{e}^{2} d z}{r},
$$

where $M(b)$ is the projected mass enclosed by the circle of the radius $b$ [9]. In another words it is the mass inside the cylinder with the radius $b$.

We have carried out the calculations for models with the nonuniform plasma distribution: singular and non-singular isothermal sphere; for hot gas inside the gravitational field of a black hole, and of a cluster of galaxies. For different gravitational lens models we compare the corrections to the vacuum lensing due to the gravity effect in plasma (second term in (4.1)), and due to the plasma inhomogeneity (third term in (4.1). We have shown that the gravitational effect could be detected in the case of a hot gas in the gravitational field of a galaxy cluster [9].

\section{Acknowledgments}

The work of GSBK and OYuT was partially supported by the Russian Foundation for Basic Research grants 08-02-00491, the RAN Program 'Origin, formation and evolution of objects of Universe' and Russian Federation President Grant for Support of Leading Scientific Schools NSh3458.2010.2.

The work of OYuT was also partially supported by the Russian Federation President Grant for Support of Young Scientists MK-8696.2010.2 and the Dynasty Foundation.

\section{References}

[1] L. D. Landau and E. M. Lifshitz, The Classical Theory of Fields, Pergamon, Oxford 1993.

[2] P. Schneider, J. Ehlers and E. Falco, Gravitational lenses (Springer-Verlag, Berlin, 1992).

[3] P. Schneider, C. S. Kochanek and J. Wambsganss, Gravitational Lensing: Strong, Weak and Micro, Swiss Society for Astrophysics and Astronomy Series: Saas-Fee Advanced Courses, Number 33, Springer, Berlin 2006. 
[4] P. V. Bliokh and A. A. Minakov, Gravitational Lenses, Naukova Dumka, Kiev 1989 (in Russian).

[5] D. O. Muhleman, R. D. Ekers and E. B. Fomalont, Phys. Rev. Lett., 24 (1970) 1377.

[6] A. P. Lightman, W. H. Press, R. H. Price and S. A. Teukolsky, Problem Book in Relativity and Gravitation, Princeton University Press, Princeton, New Jersey, New York 1979.

[7] J. L. Synge, Relativity: the General Theory, North-Holland Publishing Company, Amsterdam 1960.

[8] G. S. Bisnovatyi-Kogan and O. Yu. Tsupko, Gravitation and Cosmology 15 (2009) 20.

[9] G. S. Bisnovatyi-Kogan and O. Yu. Tsupko, MNRAS 404 (2010) 1790.

[10] V. Perlick, Ray Optics, Fermat's Principle, and Applications to General Relativity, Lecture Notes in Physics, Monographs Series, v.61, Springer-Verlag, Berlin Heidelberg New York 2000.

[11] R. Kulsrud, A. Loeb , Phys. Rev. D 45 (1992) 525.

[12] A. Broderick, R. Blandford, MNRAS 342 (2003) 1280.

[13] A. Broderick, R. Blandford, Ap\&SS 288 (2003) 161. 